\title{
Comparação entre os exames radiográficos convencional e digitalizado em relação ao plano de tratamento de superfícies oclusais
}

\section{Comparison between digitized and conventional radiography in the treatment plan for occlusal surfaces}

Dione Dias TORRIANI*

Marcelo Rodrigues GONÇALVES**

João Batista VIEIRA***

TORRIANI, D. D.; GONÇALVES, M. R.; VIEIRA, J. B. Comparação entre os exames radiográficos convencional e digitalizado em relação ao plano de tratamento de superficies oclusais. Pesqui Odontol Bras, v. 14, n. 3, p. 256-261, jul./set. 2000.

O objetivo deste estudo foi comparar as decisões de tratamento restaurador de superfícies oclusais, sem cavitação, quando realizadas através dos aspectos clínicos e radiográficos, convencional e digitalizado. Foram examinados, 33 sítios das superficies oclusais de 30 molares permanentes extraídos, com e sem pigmentação. O plano de tratamento para cada região foi realizado por 5 cirurgiões-dentistas, professores universitários, utilizando dois tipos de exames: exame visual de fotografias e radiografia interproximal convencional (IV + RXC); e exame visual de fotografias e radiografia digitalizada (IV + DIGORA). O padrão de validação para os planos de tratamento foi realizado através do aspecto histológico. A sensibilidade em determinar a não-necessidade de tratamento restaurador foi, em média, tanto para a IV + RXC quanto para a IV + DIGORA, de 0,23. A especificidade foi, em média, de 0,83 e 0,86, para a IV + RXC e IV + DIGORA, respectivamente. Quando comparou-se os planos de tratamento intra-examinadores, não foi encontrada diferença estatisticamente significante à nível de 5\%. Baseado nestes dados, pode-se concluir que os métodos radiográficos, convencional e digitalizado, não demonstraram diferenças na efetividade da determinação do plano de tratamento de superfícies oclusais sem cavitação.

UNITERMOS: Cárie dental; Radiografia dentária; Intensificação da imagem radiográfica.

\section{INTRODUÇÃO}

As lesões cariosas oclusais despertam interesse para a pesquisa devido a sua grande incidência, prevalência e dificuldades clínicas de diagnósti$\mathrm{CO}^{2,3,9,11}$ e de decisão de tratamento ${ }^{4,7,8}$.

Dentre os métodos diagnósticos mais utilizados clinicamente para estas lesões estão a inspeção visual e o exame radiográfico interproximal. Porém, diversos trabalhos ${ }^{1,10,15}$ têm demonstrado uma baixa sensibilidade ao utilizá-los.

$\mathrm{Na}$ tentativa de melhorar o diagnóstico radiográfico das lesões cariosas, através da manipulação de tamanho, brilho e contraste da imagem, o sistema de análise radiográfica por computador foi submetido à investigações científicas, objetivando o conhecimento do seu desempenho.

Estudos comparativos da literatura têm demonstrado uma tendência de maior sensibilidade dos exames radiográficos digitalizados, em relação aos convencionais ${ }^{5,6,12,16,17}$, no diagnóstico de lesões cariosas oclusais. Porém, como nem todas as lesões progridem da mesma forma, pelas peculiaridades especialmente ligadas ao paciente portador, é importante que o cirurgião-dentista não apenas diagnostique, mas, principalmente, decida sobre o tratamento.

Assim, o objetivo deste estudo foi comparar decisões de tratamento para superfícies oclusais sem

* Aluna de Pós-Graduação em Odontopediatria, em nível de Doutorado; ** Professor Assistente Doutor do Departamento de Odontologia Infantil e Social; *** Técnico em Radiologia do Departamento de Patologia e Propedêutica Clínica - Faculdade de Odontologia de Araçatuba da UNESP. 
TORRIANI, D. D.; GONÇALVES, M. R.; VIEIRA, J. B. Comparação entre os exames radiográficos convencional e digitalizado em relação ao plano de tratamento de superficies oclusais. Pesqui Odontol Bras, v. 14, n. 3, p. 256-261, jul./set. 2000.

cavitação, realizadas a partir da inspeção visual combinada ao exame radiográfico convencional e ao exame radiográfico digitalizado.

\section{MATERIAIS E MÉTODOS}

Foram examinados 33 sítios de superficies oclusais de 32 molares humanos extraídos, sem que se conhecesse as condições do meio bucal nas quais estavam expostos. Estes dentes foram mantidos hermeticamente fechados em solução de formol neutro a $2 \%$. Macroscopicamente, não apresentavam cavitação, sendo que graus variáveis de descoloração das fissuras podiam estar presentes.

Todas as superficies oclusais, após profilaxia com jatos de bicarbonato, foram identificadas numericamente e fotografadas (Medical, Nikon), obtendo-se aumento de 3 vezes. A seguir, os sítios oclusais a serem analisados foram demarcados seguindo a numeração anterior.

Para o exame radiográfico convencional, cada dente foi aderido à película radiográfica por sua porção radicular, de forma padronizada, utilizando-se cera utilidade, sendo que a superficie vestibular ficou voltada para o cilindro do aparelho de RX (Dabi Atlante, modelo Spectro II, $60 \mathrm{~Hz}, 880 \mathrm{~W}$ ). Os filmes, devidamente identificados, foram do tipo Agfa Dentus M2, de dimensões $3 \times 4 \mathrm{~cm}$. O tempo de exposição foi de $2 / 10$ de segundo, a uma distância foco-filme de $40 \mathrm{~cm}$, tendo sido o processamento radiográfico feito pelo método tempo/temperatura, utilizando-se soluções reveladora e fixadora Kodak, e secagem em secadora. A análise das imagens radiográficas convencionais foi feita com o auxílio de um negatoscópio.

Para o exame radiográfico digitalizado, a identificação, o posicionamento dos dentes sobre a película e o tempo de exposição foram os mesmos descritos anteriormente. Utilizou-se o sistema computadorizado DIGORA (Soredex, Finland), no qual realizava-se as análises das imagens sob um aumento de 4 vezes, ajustando-se o brilho e contraste.

A determinação do plano de tratamento para os sítios oclusais demarcados foi realizada por 5 professores universitários, que tomaram as suas decisões baseadas na anamnese de uma paciente (Quadro 1) e utilizando dois tipos de exames: a) inspeção visual (fotos) e exame radiográfico convencional (IV + RXC); b) inspeção visual (fotos) e exame radiográfico digitalizado (IV + DIGORA). Os examinadores não possuíam experiência na utilização do DIGORA, sendo que para as análises, re- ceberam orientações básicas sobre o seu funcionamento.

Os planos de tratamento foram anotados em fichas próprias, de acordo com três opções:

$0=$ acompanhamento

1 = selante

2 = restauração conservadora

A duplicata destes planos foi realizada dois meses após o período do primeiro exame, para determinação da concordância intra- e entre os examinadores.

\section{Processamento histológico}

Para validação dos planos de tratamento, foram obtidas duas seções no sentido buco-lingual, dos sítios demarcados anteriormente, com o auxílio de uma cortadeira Isomet (Buehler). Feitos os cortes, as seções foram removidas com auxílio de disco diamantado, montado em motor de bancada (Nevoni), e aquela que apresentava maior alteração cariosa na região de fissuras oclusais foi levada para polimento em branco de espanha e lixas d'água $n^{\circ} 400,600$ e 850, seqüencialmente. Após, os fragmentos dentários foram lavados, montados em lâminas e levados para exame em microscópio de luz refletida (Carl Zeiss, Jena).

Para a determinação da sensibilidade e especificidade dos planos de tratamento estabelecidos por cada examinador, foi convencionado um plano de tratamento "padrão ouro", seguindo os critérios mostrados no Quadro 2.

\section{Análise estatística}

Os dados foram levados para análise estatística, para fins de cálculo da sensibilidade e especificidade dos exames, em relação à decisão de tratamento dos sítios oclusais. Embora não tenha sido o objetivo deste estudo, foram calculadas as concordâncias intra-examinadores (teste kappa) e en-

QUADRO 1 - Dados da anamnese da paciente.

\begin{tabular}{||l||}
\hline \multicolumn{1}{|c||}{ Perfil da paciente } \\
\hline Sexo feminino, 15 anos de idade \\
\hline Saúde geral sem alterações \\
\hline $\begin{array}{l}\text { Família emocionalmente ajustada, integrada às atividades } \\
\text { socioculturais e nível econômico satisfatório e estável }\end{array}$ \\
\hline $\begin{array}{l}\text { Alimentação em horários regulares, com consumo de } \\
\text { açúcar restrito às refeições }\end{array}$ \\
\hline Boa higiene, sem presença de placa dental espessa \\
\hline Uso do flúor através de creme dental \\
\hline
\end{tabular}


TORRIANI, D. D.; GONÇALVES, M. R.; VIEIRA, J. B. Comparação entre os exames radiográficos convencional e digitalizado em relação ao plano de tratamento de superfícies oclusais. Pesqui Odontol Bras, v. 14, n. 3, p. 256-261, jul./set. 2000.

tre os examinadores (teste de Cochran), para verificação da reprodutibilidade dos exames.

\section{RESULTADOS}

As sensibilidades e especificidades dos planos de tratamento para as superficies oclusais, determinados pelos 5 examinadores, estão descritos nas Tabelas 1 e 2. Quando foram utilizados a inspeção visual e o exame radiográfico convencional (Tabela 1), a sensibilidade variou entre $0 \%$ a $50 \%$, sendo que a média obtida foi de $23 \%$, e a especificidade variou entre $66 \%$ e $100 \%$, sendo que a média foi $83 \%$. Quando os examinadores fizeram uso da inspeção visual e do exame radiográfico digitalizado (Tabela 2), obteve-se uma variação na sensibilidade entre $0 \%$ e $33 \%$, com média de $23 \%$. A especificidade variou entre $70 \%$ e $100 \%$, obtendo-se uma média de $86 \%$.

O examinador 2 determinou que todos os dentes observados receberiam como tratamento o acompanhamento. Este observador obteve sensibilidade de $0 \%$ e especificidade de $100 \%$ para ambos os exames. O observador 4, o qual obteve $50 \%$ de sensibilidade no método da IV + RXC, atingiu, porém, a menor especificidade (66\%). Este mesmo observador encontrou valores bem diferentes ao determinar seus planos de tratamento com a

QUADRO 2 - Escore binário dos planos de tratamento.

\begin{tabular}{|c|c||}
\hline Plano de tratamento & Validação histológica \\
\hline $\begin{array}{c}\text { não-invasivo }=>\text { escore } 0 \\
\text { (acompanhamento e selante) }\end{array}$ & sem alteração em dentina \\
\hline $\begin{array}{c}\text { invasivo }=>\text { escore } 1 \\
\text { (restauração conservadora })\end{array}$ & com alteração em dentina \\
\hline \hline
\end{tabular}

TABELA 1 - Sensibilidade e especificidade média da inspeção visual e exame radiográfico convencional, relacionadas ao plano de tratamento das superficies oclusais.

\begin{tabular}{c|c|c}
\hline \hline \multirow{2}{*}{ Examinadores } & Sensibilidade & Especificidade \\
\cline { 2 - 3 } & $\%$ & $\%$ \\
\hline 1 & 0 & 92 \\
\hline 2 & 0 & 100 \\
\hline 3 & 33 & 81 \\
\hline 4 & 50 & 66 \\
\hline 5 & 33 & 78 \\
\hline Média & 23 & 83 \\
\hline \hline
\end{tabular}

IV + DIGORA (sensibilidade de $17 \%$ e especificidade de $70 \%$ ), sendo, novamente, o menor valor de especificidade de todos os observadores.

A Tabela 3 mostra os valores de concordância inter- e intra-examinadores quando realizados os dois tipos de exames. Observamos que a concordância interexaminadores não foi estatisticamente significante ao nivel de $5 \%$ em ambos os métodos, e quando comparados, os valores foram semelhantes. Quanto à concordância intra-examinadores, os valores encontrados foram bons frente aos dois exames, alcançando os valores máximos (100\%) com o examinador 2 , e os menores valores com o examinador 4 (67\% e $76 \%)$.

\section{DISCUSSÃO}

O exame radiográfico tem sido apontado como um método complementar praticamente indispensável no auxílio ao diagnóstico e tratamento de lesões de cárie em superfícies oclusais. Portanto, neste estudo, foram feitas comparações entre os planos de tratamento para superficies oclusais obtidos a partir de dois métodos radiográficos, o con-

TABELA 2 - Sensibilidade e especificidade média da inspeção visual e exame radiográfico digitalizado, relacionadas ao plano de tratamento das superficies oclusais.

\begin{tabular}{c|c|c}
\hline \hline \multirow{2}{*}{ Examinadores } & Sensibilidade & Especificidade \\
\cline { 2 - 3 } & $\%$ & $\%$ \\
\hline 1 & 33 & 89 \\
\hline 2 & 0 & 100 \\
\hline 3 & 33 & 88 \\
\hline 4 & 17 & 70 \\
\hline 5 & 33 & 81 \\
\hline Média & 23 & 86 \\
\hline \hline
\end{tabular}

TABELA 3 - Concordância intra- e interexaminadores em relação aos planos de tratamento das superficies oclusais, utilizando os dois tipos de exame.

\begin{tabular}{c|c|c|c|c|c|c|c}
\hline \multirow{2}{*}{ Exames } & \multicolumn{5}{|c|}{ Intra-examinadores } & \multicolumn{1}{c}{$\begin{array}{c}\text { Interexa- } \\
\text { minadores }\end{array}$} \\
\cline { 2 - 8 } & \multicolumn{6}{c|}{ (Teste kappa) } & \multicolumn{2}{c}{$\begin{array}{c}\text { (Teste } \\
\text { Cochran) }\end{array}$} \\
\hline \multirow{2}{*}{ IV + RXC } & 1 & 2 & 3 & 4 & 5 & $X^{2}$ & $\%$ \\
\hline IV + DIGORA & 94 & 100 & 88 & 67 & 85 & $67,09 *$ & 0,03 \\
\hline \hline
\end{tabular}

*não significativa estatisticamente ao nível de $5 \%$. 
TORRIANI, D. D.; GONÇALVES, M. R.; VIEIRA, J. B. Comparação entre os exames radiográficos convencional e digitalizado em relação ao plano de tratamento de superficies oclusais. Pesqui Odontol Bras, v. 14, n. 3, p. 256-261, jul./set. 2000.

vencional e o digitalizado, associados a uma inspeção visual feita a partir de fotografias destes sítios, considerando-se, também, o perfil de uma paciente.

$\mathrm{Na}$ presente investigação, os observadores, todos cirurgiões-dentistas e professores universitários e, portanto, formadores de opinião e responsáveis pela divulgação e interpretação de correntes científicas, tomavam a decisão por um tratamento invasivo ou não, em função dos aspectos clínico e radiográfico e dos dados da anamnese descritos.

Para a determinação dos valores de sensibilidade e especificidade, o conceito utilizado não foi aquele relacionado com a capacidade dos métodos em diagnosticar a presença ou ausência da doença, mas sim, com a capacidade dos mesmos em determinar a necessidade de tratamento invasivo quando ele realmente fosse necessário (sensibilidade), e a capacidade de determinar a não-necessidade de tratamento invasivo quando ele realmente fosse desnecessário (especificidade).

A comparação entre os valores médios de sensibilidade e especificidade dos planos de tratamento feitos com os dois tipos de exames $(23 \%$ e $83 \%$ no IV + RXC e $23 \%$ e $86 \%$ no IV + DIGORA, respectivamente) não demonstraram diferenças estatísticas entre ambos.

Nos estudos em que se comparou a sensibilidade e a especificidade do exame radiográfico convencional com o digitalizado, foi verificado uma tendência de melhor desempenho do método radiográfico digitalizado em relação ao convencional, quanto ao diagnóstico de lesões cariosas oclusais $^{5,6,12,16}$. Porém, podemos observar no presente estudo que, em relação ao plano de tratamento de superficies oclusais, isto não teve grande influência, pois os métodos clínicos de diagnóstico são apenas formas de se obter informações necessárias para determinar-se o plano de tratamento, devendo considerar, também, fatores interferentes ligados ao paciente e ao profissional (TORRIANI; ROMANO $\left.^{14}, 1997\right)$.

KAY; NUTTALL ${ }^{8}$ (1995) sugeriram um diagrama para designar a necessidade ou não de tratamento invasivo (Figura 1). A parte deste diagrama de cor preta, representaria os casos que requerem tratamento restaurador; a parte de cor branca, caracterizando a saúde, sinalizaria os casos que não requerem, ficando, assim, a cor cinza representante da "zona de transição". Dentro deste diagrama, cada profissional determina seu "ponto limite", o qual é definido como o ponto de decisão da realização, ou não, do tratamento restaurador. E este ponto pode variar de acordo com vários fatores, ligados ao paciente e ao profissional.

Considerando este diagrama, podemos verificar que o observador 4, comparativamente, posicionou-se mais para a zona branca (especificidades de $66 \%$ e $70 \%$ ), obtendo um maior número de casos em que os dentes foram restaurados desnecessariamente, ou seja, incorreu em sobretratamento. Analisando o mesmo observador, verificou-se que apesar de ter atingido o maior valor de sensibilidade com o método da IV + RXC (50\%), atingiu a menor especificidade.

O observador 2 obteve, nos dois exames, a menor sensibilidade $(0 \%)$, ao mesmo tempo em que obteve a maior especificidade (100\%). Isto significa que este observador não teria realizado o tratamento restaurador nas situações indicadas por nós, ou seja, quando houvesse envolvimento dentinário, submetendo todos os pacientes à rotina de acompanhamento. Entretanto, não teria cometido nenhum caso de sobretratamento, pois todos os seus tratamentos iniciais seriam reversiveis.

O cirurgião-dentista, ao tomar uma decisão de tratamento, deve decidir por qual oferecerá menores possibilidades de erro, ou seja, qual estará mais próxima da zona preta e qual aproximar-se-á mais da zona branca. De acordo com THYLSTRUP; FEJERSKOV ${ }^{13}$ (1997), o cirurgião-dentista desempenha papel preponderante no planejamento do tratamento dos pacientes, tendo em vista que o sucesso no plano depende do seu conhecimento científico sobre cárie e de como estas informações são transmitidas aos pacientes. A tomada de decisão para tratamento de lesões cariosas é, portanto, contextual, dependente dos pontos levantados acima. Isto ficou claro após análise das decisões de tratamento do observador 4, o qual apresentou menores valores de concordância intra-examinador, demonstrando que, na realidade, os profissionais estão sujeitos a mudanças ou diferenças, tanto por ausência de critérios rígidos quanto por aquisição de novos conhecimentos em relação à prática odontológica.

Neste estudo, a sensibilidade e especificidade das decisões para intervenção foram determinadas em função de um plano de tratamento "padrão

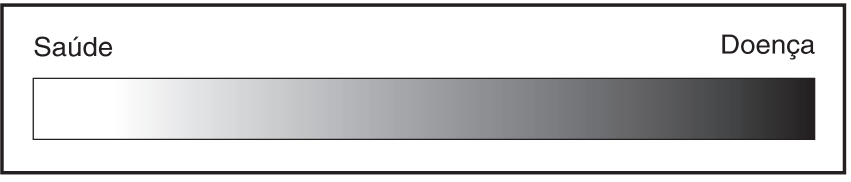

FIGURA 1 - Zonas limites de tratamento (KAY; NUTTALL $\left.{ }^{8}, 1995\right)$. 
TORRIANI, D. D.; GONÇALVES, M. R.; VIEIRA, J. B. Comparação entre os exames radiográficos convencional e digitalizado em relação ao plano de tratamento de superfícies oclusais. Pesqui Odontol Bras, v. 14, n. 3, p. 256-261, jul./set. 2000.

ouro", em que se convencionou que, havendo comprometimento de dentina, deveria haver tratamento invasivo, ou seja, restauração conservadora. Este parâmetro foi estabelecido com o intuito de medir possiveis mudanças de atitudes dos dentistas com o auxílio de um método ou de outro, e não com a finalidade de afirmar que, frente a estas situações na clínica, optariamos por um tratamento invasivo. Como dito acima, decisão de tratamento é mais do que diagnóstico de presença ou não de lesão cariosa, representa a ação do dentista, associando seus conhecimentos à postura clínica.

Acreditamos que o presente estudo traz informações novas em relação à utilização de métodos radiográficos digitalizados como auxiliar no planejamento do melhor tratamento clínico das lesões cariosas oclusais. É comum os trabalhos existentes na literatura ${ }^{5,12,17}$ fazerem uma abordagem comparativa entre os exames radiográficos convencionais e digitalizados quando utilizados no diagnóstico das lesões cariosas, porém, não encontramos, até o momento, trabalhos que comparassem estes métodos quando usados no auxílio do plano de tratamento. Por sua vez, as estratégias de tratamento de lesões cariosas oclusais, quando estudadas e analisadas, têm sido relacionadas com a inspeção visual e radiografia convencional, de maneira isolada ou associada ${ }^{4,7,8}$. Portanto, de acordo com a metodologia empregada e as situações disponiveis, os resultados aqui encontrados nos dão segurança para dizer que para a referida finalidade, os dois métodos apresentam efetividade semelhante, sendo que, a escolha de um ou outro, não interfere decisivamente na terapia a ser instituída para as lesões cariosas oclusais. Este dado é de grande importância para a época atual, na qual os grandes avanços tecnológicos procuram criar uma demanda de consumo, e muitas vezes, o profissional clínico sem maiores condições de análise crítica, pode ser levado a desenvolver um conceito impróprio sobre a utilização destas tecnologias.

\section{CONCLUSÃO}

Através do presente estudo pôde-se concluir que:

- os métodos radiográficos convencional e digitalizado, utilizados juntamente com a inspeção visual (foto), não apresentaram diferenças estatisticamente significantes na determinação do plano de tratamento de lesões cariosas oclusais;

- a radiografia digitalizada, a qual envolve uma tecnologia de alto custo para o profissional, pode ser substituída pela radiografia convencional no plano de tratamento das lesões oclusais;

- a concordância intra- e interexaminadores, de uma maneira geral, foram satisfatórias.

TORRIANI, D. D.; GONÇALVES, M. R.; VIEIRA, J. B. Comparison between digitized and conventional radiography in the treatment plan for occlusal surfaces. Pesqui Odontol Bras, v. 14, n. 3, p. 256-261, jul./set. 2000.

The objective of this study was to compare the treatment plan decisions made for intact occlusal surfaces using different diagnostic tools. Clinical photographs and either conventional or digitized radiographs were used to evaluate 33 regions of the occlusal surfaces of 30 extracted permanent molars. The teeth had regions with and without pigmentation. The treatment plan decisions were made by 5 dental faculty members using the two examination methods: 1$)$ visual exam of photographs and conventional radiographs (IV+RXC); and 2) visual exam of photographs and digitized radiographs (IV+DIGORA). The regions were assigned a value of 0 (no treatment needed), 1 (need for sealant but not for restoration), and 2 (need for restoration). The validation of the treatment plans was accomplished through histological examination of tooth sections from each of the regions. The results were analyzed using a Cochran test and a sign test. The sensitivity, determining what percentage of the teeth needed treatment, was very low (23\%) for both IV+RXC and IV+DIGORA. The specificity, determining the percentage of teeth with no disease, was much higher: $83 \%$ and $86 \%$ for IV+RXC and IV+DIGORA, respectively. There was no significant difference in the tratment plan decisions made with either of the diagnostic tools $(\mathrm{p}<0.05)$. Based on these data, it can be concluded that both conventional and digitized radiographic methods led to similar treatment plan decisions for intact occlusal surfaces.

UNITERMS: Dental caries; Radiography, dental; Radiographic image enhancement. 
TORRIANI, D. D.; GONÇALVES, M. R.; VIEIRA, J. B. Comparação entre os exames radiográficos convencional e digitalizado em relação ao plano de tratamento de superficies oclusais. Pesqui Odontol Bras, v. 14, n. 3, p. 256-261, jul./set. 2000.

\section{REFERÊNCIAS BIBLIOGRÁFICAS}

1. CREANOR, S. L.; RUSSELL, J. I.; STRANG, D. M.; STEPHEN, K. W.; BURCHELL, C. K. The prevalence of clinically undetected occlusal dentine caries in Scottish adolescents. Br Dent J, v. 169, p. 126-129, 1990.

2. DOWNER, M. C. Concurrent validity of epidemiological diagnostic system for caries with histological appearance of extracted teeth as validating criterion. Caries Res, v. 9, p. 231-246, 1975.

3. FLAITZ, C. M.; HICKS, M. J.; SILVERSTONE, L. M. Radiographic, histological and eletronic comparison of occlusal caries: an in vivo study. Pediat Dent, v. 8, p. 24-28, 1986.

4. GRÖNDAHL, H. G. Decisions strategies in radiographic caries diagnosis. Swed Dent J, v. 3, p. 173-180, 1979.

5. HINTZE, H. Screening with conventional and digital bite-wing radiography compared to clinical examination alone for caries detection in low-risk children. Caries Res, v. 27, p. 499-504, 1993.

6. HUYSMANS, M. C. D.; LONGBOTTOM, C.; PITTS, N. B. In vitro comparison of six diagnostic methods for occlusal caries, including two electrical techniques. Caries Res, v. 30, p. 273, 1996. (Abstract 18).

7. KAY, E. J.; NUTTALL, N. M. Relationship between dentists' treatment attitudes and restorative decisions made on the basis of simulated bitewing radiographs. Community Dent Oral Epidemiol, v. 22, p. 71-74, 1994.

8. KAY, E. J.; NUTTALL, N. Clinical decision making - an art or a science? Part III: to treat or not to treat? Brit Dent J, v. 178, p. 153-155, 1995.

9. KRAMER, P. F.; FELDENS, C. A.; ROMANO, A. R. Promoção de saúde bucal em Odontopediatria: diagnóstico, prevenção e tratamento da cárie oclusal. São Paulo : Artes Médicas, 1997. 144 p.

10. NYTUN, R. B.; RAADAL, M.; ESPELID, I. Diagnosis of dentin involvement in occlusal caries based on visual and radiographic examination of the teeth. Scand $\mathbf{J}$ Dent Res, v. 100, p. 114-118, 1992.

11. RIPA, L. W.; LESKE, G. S.; VARMA, A. O. Longitudinal study of the caries susceptibility of occlusal and proximal surfaces of first permanent molars. J Public Health Dent, v. 48, p. 8-13, 1988.

12. RUSSEL, M.; PITTS, N. B. Radiovisiograph diagnosis of dental caries: initial comparison of basic mode videoprints with bitewing radiography. Caries Res, v. 30, p. 270, 1996. (Abstract 12).

13. THYLSTRUP, A.; FEJERSKOV, O. Diagnóstico radiológico no tratamento da cárie dentária. In: GRÖNDAHL, H. J. Cariologia clínica. São Paulo : Livraria Santos, 1997. p. 367-380.

14. TORRIANI, D. D.; ROMANO, A. R. Plano de tratamento. In: BUSATO, A. L. S.; BARBOSA, A. N.; BUENO, M.; BALDISSERA, R. A. Dentística. Restaurações em dentes anteriores. São Paulo : Artes Médicas, 1997. p. 25-32.

15. VAN AMEROGEN, J. P.; PENNING, C.; KIDD, E. A. M.; TEN CATE, J. M. An in vitro assessment of the extent of caries under small occlusal cavities. Caries Res, v. 26, p. 89-93, 1992.

16. WENZEL, A.; FEJERSKOV, O. Validity of diagnosis of questionable caries lesions in occlusal surfaces of extracted third molars. Caries Res, v. 26, p. 188-194, 1992.

17. WENZEL, A.; LARSEN, M. J.; FEJERSKOV, O. Detection of occlusal caries without cavitation by visual inspection, film radiographs, xeroradiographs and digitized radiographs. Caries Res, v. 25, p. 365-371, 1991.
Recebido para publicação em 04/01/00

Enviado para reformulação em 14/03/00

Aceito para publicação em 24/05/00 